\title{
Programas sociales y su incidencia en reducción de pobreza en un asentamiento humano, Perú, 2018
}

\section{Social programs and their incidence in reducing poverty in a Human Settlement, Peru, 2018}

Alex Javier Sánchez Pantaleón ${ }^{1}$, Rosa Ysabel Bazán Valque², Benjamín Roldan Polo Escobar ${ }^{3}$

\section{RESUMEN}

La investigación tuvo como objetivo determinar el nivel de incidencia de los programas sociales Comedor Popular, Vaso de Leche y Cuna Más, en la reducción de la pobreza de los beneficiarios en el Asentamiento Humano Pedro Ruiz Gallo de Lambayeque en el año 2018. El estudio fue de enfoque cuantitativo, descriptivo, no experimental. El universo muestral fue 137 beneficiarios de los programas sociales y se aplicó como instrumento la encuesta. Los resultados indican que la mayoría de encuestados (100\%), de Cuna Más y Comedor Popular sí ayudaron a reducir su pobreza y por el contrario el $72 \%$ de beneficiarios de Vaso de Leche manifestaron que no. Se concluyó que el programa Cuna Más es el más valorado, con calificación de bueno $73 \%$ y excelente $25 \%$. Vaso de Leche es el único que el 72\% indicaron que no les ha ayudado a la reducción de su pobreza. Los beneficiarios que continuaran hasta que se les retire el beneficio son: Cuna Más 90\%, Comedor Popular 50\%, Vaso de leche $88 \%$. Se concluye que la pobreza por necesidades básicas insatisfechas es alta, 94,4 \% en la deficiencia de servicios básicos y en vivienda $58,4 \%$. La pobreza monetaria es alta con $58,4 \%$ en la clasificación pobre y $23,4 \%$ como pobre extremo.

Palabras clave: : Políticas públicas, pobreza, programas sociales.

\begin{abstract}
The objective of the research was to determine the level of incidence of the Comedor Popular, Vaso de Leche and Cuna Más social programs in reducing the poverty of the beneficiaries in the Pedro Ruiz Gallo, human settlement in Lambayeque, in 2018. The study was quantitative, descriptive, not experimental. The sample universe was 137 beneficiaries of social programs and the survey was applied as an instrument. The results indicate that the majority of respondents (100\%), from Cuna Más and Comedor Popular did help reduce their poverty and, on the contrary, $72 \%$ of beneficiaries from Vaso de Leche said they did not. It was concluded that the Cuna Más program is the most valued, with a rating of $73 \%$ good and $25 \%$ excellent. Glass of Milk is the only one that $72 \%$ indicated that it has not helped them reduce their poverty. The beneficiaries that will continue until the benefit is withdrawn are: Cuna Más 90\%, Comedor Popular 50\%, Vaso de leche 88\% Poverty due to unsatisfied basic needs is high $94.4 \%$ in the deficiency of basic services and housing $58.4 \%$. Monetary poverty is high with $58.4 \%$ classified as poor and $23.4 \%$ as extreme poor.
\end{abstract}

Keywords:Public policies, poverty, social programs.

\footnotetext{
${ }^{1} 1$ Maestro en Gestión Pública. Docente en la Facultad de Ciencias Económicas y Administrativas perteneciente a la Escuela Profesional de Economía de la Universidad Nacional Toribio Rodríguez de Mendoza de Amazonas. Correo electrónico: alex.sanchez@untrm.edu.pe

2 Doctora en Gestión Pública y Gobernabilidad .Docente en la Facultad de Ciencias Económicas y Administrativas perteneciente a la Escuela Profesional de Administración de Empresas de la Universidad Nacional Toribio Rodríguez de Mendoza de Amazonas. Correo electrónico: rosa.bazan@untrm.edu.pe

3 Doctor en Gestión Pública y Gobernabilidad. Docente en la Facultad de Ciencias Económicas y Administrativas perteneciente a la Escuela Profesional de Economía de la Universidad Nacional Toribio Rodríguez de Mendoza de Amazonas. Correo electrónico: brpoloescobar@gmail.com
} 


\section{I.INTRODUCCIÓN}

Los gobiernos de los distintos países en el mundo, tienen la obligación de crear mejores condiciones de vida a su población, por lo cual la pobreza es un problema que se viene combatiendo durante muchos años y para su erradicación se han implementado diferentes políticas públicas como los programas sociales, con objetivos a corto y largo plazo. Pero no todas las políticas puestas en marcha han logrado los objetivos esperados, muchos de ellos no son sostenibles en el tiempo, y teniendo en cuenta que los recursos de cada gobierno son limitados, se hace necesaria una evaluación constante de los impactos de estas políticas públicas, para no desperdiciar los recursos escasos y aprovecharlos de la mejor manera en beneficio de la población.

La erradicación de la pobreza es el objetivo común de diferentes gobiernos y organizaciones en el mundo, como la Organización de las Naciones Unidas, su objetivo es "erradicar la pobreza absoluta y reducir sustancialmente la pobreza en el mundo mediante la acción decisiva de cada país y la cooperación internacional" (ONU, 2017, párr.1). Para el Banco Mundial (2017) uno de sus objetivos primordiales para el 2030, es la erradicación de la pobreza extrema (p.1). En los cambios actuales del Estado de Bienestar, la intervención en materia de políticas sociales ofreció un lugar al ciudadano, pero también obligó al Estado a proveer asistencia y ser el soporte de todo aquel que tuviese necesidades como consecuencia de las transformaciones económicas.

Según la ONU (2017) en 2013, unos 767 millones de personas vivían por debajo del umbral de pobreza internacional, con 1,90 dólares diarios, frente a 1700 millones de personas en 1999. Estos datos muestran que la tasa se redujo en un $16 \%$, pero no es suficiente. El lugar donde más disminuyó la pobreza fue en Asia Oriental y Suroriental, donde la tasa se redujo en $32 \%$ de 1999 al 2013. Sin embargo, el 42\% de las personas de África Subsahariana seguían viviendo en condiciones de pobreza extrema en 2013 (párr.5).

De acuerdo a RT (2016) unos 1000 millones de niños en todo el mundo aún se encuentran en pobreza. Según los datos de Fondo de las Naciones Unidas para la Infancia (UNICEF), 22.000 niños mueren cada día a causa de la pobreza y en todo el mundo hay 805 millones de personas que pasan hambre (párr.5).

El Programa de las Naciones Unidas para el Desarrollo (2016) sostiene que la desigualdad en la distribución y control de los recursos políticos, económicos y sociales, así como las instituciones sociales discriminatorias que incrementan el ciclo de exclusión son factores que perpetúan la desigualdad y pobreza por generaciones (párr.4).

De acuerdo a la Comisión Económica para América Latina y el Caribe (2017) en los últimos 10 años, los países de América Latina y el Caribe han mejorado gracias a la implementación de políticas y programas que permiten lograr mayor inclusión social y laboral de la población, pero estos progresos son insuficientes para alcanzar el desarrollo con igualdad y sostenibilidad existiendo un riesgo de retroceso en la actual coyuntura de bajo crecimiento de las economías de cada país (párr.2).

Según informe de la CEPAL (2017) en 2014, el 28,5\% de la población de América Latina era pobre, sin embargo en el 2016 el porcentaje aumentó hasta el $30,7 \%$. Asimismo durante este mismo periodo de tiempo, la pobreza extrema subió de $9 \%$ a $10,2 \%$. Esta situación de debió a la desaceleración del crecimiento de la economía regional y aumento de la pobreza en Brasil y Venezuela (párr.2).

De acuerdo a la Encuesta Nacional de Condiciones de Vida de la Población Venezolana (2017) la pobreza por ingresos aumentó $5,2 \%$ en un año y pasó de $81,8 \%$ en 2016 a $87 \%$ en 2017 . Eso quiere decir que casi 9 de cada 10 hogares venezolanos no tienen el dinero para acceder a los bienes mínimos necesarios para vivir. Seis de cada 10 venezolanos $(59,6 \%)$ dicen ser beneficiarios de algún programa social, es decir, 13,4 millones de personas, de los cuales el 94\% recibe atención de los programas de alimentación (párr.7).

Según el informe del Instituto Nacional de Estadística e Informática (2016) el Perú redujo considerablemente la pobreza monetaria a nivel nacional, pasando de $49,1 \%$ en el 2006 a solo $20,7 \%$ en el 2016, es decir se redujo 28,4 puntos porcentuales (7 millones 304 mil dejaron de ser pobres), Cajamarca y Huancavelica son los departamentos más pobres del Perú en el 2016, con una pobreza mayor a $48,8 \%$. Sin embargo la pobreza medida por las necesidades básicas insatisfechas bajaron solo de $31 \%$ a $18 \%$ en este mismo periodo, es decir, mientras la pobreza monetaria se redujo más de la mitad, las necesidades básicas insatisfechas se redujeron menos de la mitad. Según la República (2017) en la entrevista al economista de Oxfam Armando Mendoza dijo que la tasa de la reducción de la pobreza se está desacelerando y hay una relación directa con el menor crecimiento de la economía. "En épocas de alto crecimiento económico y de generación de empleo, los programas sociales jugaban un papel menor, pero ahora esta situación se ha invertido, el rol que juegan los programas sociales es fundamental" (párr.11). 
Según el INEI (2016) la región Lambayeque tiene una tasa de pobreza monetaria entre $14 \%$ a $18,1 \%$ junto a Junín y Tacna (p.21).De acuerdo a el diario El Informante (2016) entrevistó a la directora del INEI, Rosa Nisama y sostuvo que los distritos con más índice de pobreza de la región son Cañaris, Mórrope, Chóchope, Incahuasi", con una gran demanda de servicios básicos insatisfechos (párr.1).De acuerdo al Ministerio de la mujer y poblaciones vulnerables (2015) en la Región Lambayecana funciona el Programa Integral Nacional para el Bienestar Familiar-INABIF,el consejo nacional para la integración de las personas con discapacidad CONADIS y el programa nacional YACHAY - PNY que ha brindado atención integral a niñas, niños y adolescentes que se encuentran en situación de calle, interviniendo en la provincia de Chiclayo, en los distritos de Chiclayo y José Leonardo Ortiz (p.3). Otros programas sociales presentes en la región son Pensión 65, Cuna Mass, Juntos y QaliWarma.

Por la presencia de pobreza en el A.H. Pedro Ruiz Gallo, ubicado en el distrito, provincia y región Lambayeque existen algunos programas sociales en el sector como Cuna Más, Vaso de Leche y Comedor Popular, pero que solo atienden una pequeña parte de la población.

El problema quedó formulado en los siguientes términos ¿Cúal ha sido la incidencia de los programas sociales en la reducción de la pobreza en el Asentamiento Humano "Pedro Ruíz Gallo", de Lambayeque?

Por lo cual el objetivo general fue determinar la incidencia de los programas sociales Comedor Popular, Vaso de Leche y Cuna Más, en la reducción de la pobreza de los beneficiarios en el A.H. Pedro Ruiz Gallo de Lambayeque.

Los objetivos específicos fueron: a) Determinar la valoración de los programas sociales por parte de los beneficiarios. b) Determinar la percepción de ayuda de los programas sociales en la reducción de pobreza. c) Determinar la continuidad de los beneficiarios en los programas sociales. d) Determinar la pobreza por necesidades básicas insatisfechas y pobreza monetaria de los beneficiarios de los programas sociales.

Para el estudio se encuentran antecedentes como:

Girbés (2014) en su investigación "El Contrato de inclusión dialógica: una actuación de éxito en la superación de la pobreza y la exclusión social en contextos urbanos"; concluyó que, la participación de la comunidad en la toma de las decisiones ha posibilitado la activación de acciones que realmente responden a sus necesidades. Como resultado, los planteamientos, los procesos y las acciones emprendidas son sensibles y tienen en cuenta las características raciales, lingüísticas o culturales de las minorías como por ejemplo el pueblo gitano en el barrio de La Milagrosa o las personas inmigrantes en el barrio Montserrat (p.336).

Muñoz (2016) en su investigación, "Implementación de políticas de superación de la pobreza en Chile 1990-2010. Caso Fosis", concluyó que los altos niveles de pobreza, dan cuenta que las políticas sociales tradicionales no han sido suficientes para poder reducir la pobreza, lo que lleva a buscar nuevas formas de enfrentar el tema de la pobreza, con una mirada integral (p.354).

Las políticas públicas se les considera como el conjunto de objetivos, decisiones y acciones que lleva a cabo un gobierno que goza de autoridad y legitimidad gubernamental para solucionar los problemas o asuntos públicos que en un momento determinado los ciudadanos y el propio gobierno consideran prioritarios (Bugallo,2015, párr.2).

El portal DePerú (2018) señala que "los programas sociales son actividades o instituciones creadas para apoyar y ayudar a la población de bajos recursos económicos en diversas formas que permitan mejorar la calidad de vida de las personas" (párr.2).

El Ministerio de Economía y Finanzas (2018) considera que un programa social puede ser universal como por ejemplo la educación inicial, primaria y secundaria o salud básica. Pero también pueden ser programas focalizados que atiende a determinado grupo, región o problema y se implementa empleando focalización geográfica o individual, como es el caso del Seguro Integral de Salud (SIS), el Programa del Vaso de Leche, el Programa de Comedor Popular, los programas de alimentación y nutrición de PRONAA, el Programa Juntos, los Programas de Inversión Social y Productiva, de Provías Descentralizado, FONCODES, AGRORURAL y Electrificación Rural, entre otros (párr.3).

La pobreza es una condición de privación para acceder a una canasta de bienes y servicios básicos con los cuales se pueda vivir adecuadamente en términos de los estándares prevalecientes de necesidades y satisfactores, determinados por una sociedad de acuerdo a su nivel de desarrollo (INEI, 2017, párr.4).

El INEI (2017) señala que la línea de pobreza es el valor monetario con el cual se muestra y compara el gasto per cápita mensual de un hogar para determinar si está en condiciones debe ser considerarlo pobre o no. El método de la línea de la pobreza utiliza una 
canasta de bienes y servicios (canasta normativa de satisfactores esenciales), cuyo valor per cápita (línea de pobreza) es equivalente al mínimo necesario para la sobrevivencia humana. Define a la población en situación de pobreza como aquel conjunto de personas cuyo nivel de bienestar, expresado en valor monetario, es inferior a la línea de pobreza. Para el año 2016 la línea de pobreza en la costa urbana en el Perú, es de S/ 326, es decir si el gasto per cápita (por persona), es menor a este monto, la persona será considera pobre. Por otro lado si el gasto per cápita es menor a 176 será considerada como pobre extremo (p.33-38).

Según el Instituto de Estadística y Censos, INEC (2018) es una medida de pobreza multidimensional desarrollada en los 80's por la Comisión Económica para América Latina y el Caribe (CEPAL). El método abarca cinco dimensiones y dentro de cada dimensión existe indicadores que miden privaciones, y son las siguientes que de cumplirse se les considerará pobres : capacidad económica (si los años de escolaridad del jefe de hogar, es igual o menor a 2 años y si existen más de 3 personas por cada persona ocupada en el hogar), acceso a educación básica (si existen en el hogar niños de 6 a 12 años de edad que no asisten a clases), acceso a vivienda(si el material del piso es de tierra u otros materiales no resistentes, el material de las paredes son de caña, esteras u otros similares), acceso a servicios básicos (no tiene servicios higiénicos, usa letrina o pozo ciego, no tiene conexión de agua de una red pública), hacinamiento (si hay más de 3 personas por dormitorio) (párr.1).

Teniendo en cuenta que uno de los objetivos primordiales del Perú es la reducción de la pobreza y mejorar la calidad de vida de millones de peruanos es relevante toda investigación que contribuya en el bienestar de todos los ciudadanos. Por ello esta investigación aporta resultados desde la visión cercana de un A.H. que es la realidad que sufren la mayoría de las regiones a nivel nacional y que el Estado necesita con urgencia seguir implementando acciones de mejora.

\section{MATERIAL Y MÉTODO}

La investigación fue de tipo descriptiva, no experimental, de enfoque cuantitativo.

El universo muestral fue de 137 beneficiarios de los programas sociales del Asentamiento Humano Pedro Ruiz Gallo de Lambayeque, como se detalla a continuación: 40 beneficiarios del Vaso de Leche, de los cuales todos fueron niños con edades entre 0 y 13 años. Asimismo en Cuna Más fueron 52 madres beneficiarias (los niños atendidos están entre las edades entre 0 y 3 años). El Comedor Popular con 25 niños (de 0 a 13 años de edad), 10 adultos (de 30 a 45 años) y 5 ancianos (mayores a 60 años) que reciben asistencia alimentaria.

Se aplicó una encuesta a los 137 beneficiarios de los tres programas sociales, Cuna Más, Comedor Popular y Vaso de Leche del A.H. Pedro Ruiz Gallo, la validación del instrumento se realizó con participación de tres expertos.

Los programas sociales considerados en el estudio hasta el 2018, año en que se desarrolla el estudio tienen la siguiente antigüedad: Vaso de Leche y Comedor Popular 10 años. Cuna Más 1 año.

Se especifica que los beneficiarios encuestados en el estudio tienen distintos tiempos de permanencia, según los registros son: En el programa Vaso de Leche de sus 40 beneficiados, 30 llevan de 1 a 3 años en el programa y 10 integrantes restantes más de 3 años. En el Comedor Popular de sus 40 beneficiarios, 34 llevan de 1 a 4 años, 5 de 3 a 6 años y 1 más de 6 años. En Cuna Más de 57 beneficiarios, todos llevan menos de un año ya que es un programa que lleva poco tiempo implementado.

\section{RESULTADOS}

Tabla 1. Porcentaje de la calificación por parte de los beneficiarios hacia los programas, Cuna Más, Comedor Populary Vaso de Leche.

\begin{tabular}{lccc}
\hline $\begin{array}{l}\text { Calificación } \\
\text { para los } \\
\text { programas } \\
\begin{array}{l}\text { sociales por los } \\
\text { beneficiarios }\end{array}\end{array}$ & Cuna Más & $\begin{array}{l}\text { Comedor } \\
\text { popular }\end{array}$ & Vaso de leche \\
\hline Excelente & $25 \%$ & $9 \%$ & $0 \%$ \\
Bueno & $73 \%$ & $87 \%$ & $0 \%$ \\
Regular & $2 \%$ & $4 \%$ & $68 \%$ \\
Insuficiente & $0 \%$ & $0 \%$ & $30 \%$ \\
Deficiente & $0 \%$ & $0 \%$ & $2 \%$ \\
\hline Total & $100 \%$ & $100 \%$ & $100 \%$ \\
\hline
\end{tabular}

Tabla 2. Porcentaje de los beneficiarios que opinan si ha mejorado su condición de pobreza por los programas, Cuna Más, Comedor Popular, Vaso de Leche.

\begin{tabular}{llll}
\hline $\begin{array}{l}\text { Opinión de los } \\
\text { beneficiarios si } \\
\text { ha mejorado su } \\
\begin{array}{l}\text { condición de } \\
\text { pobreza }\end{array}\end{array}$ & Cuna Más & $\begin{array}{l}\text { Comedor } \\
\text { popular }\end{array}$ & Vaso de leche \\
\hline $\mathrm{Si}$ & $100 \%$ & $100 \%$ & $28 \%$ \\
No & 0 & 0 & $72 \%$ \\
\hline Total & $100 \%$ & $100 \%$ & $100 \%$ \\
\hline
\end{tabular}


Tabla 3.Porcentaje de hasta cuándo los beneficiarios seguirán en los programas, Cuna Más, Comedor Popular, Vaso de Leche.

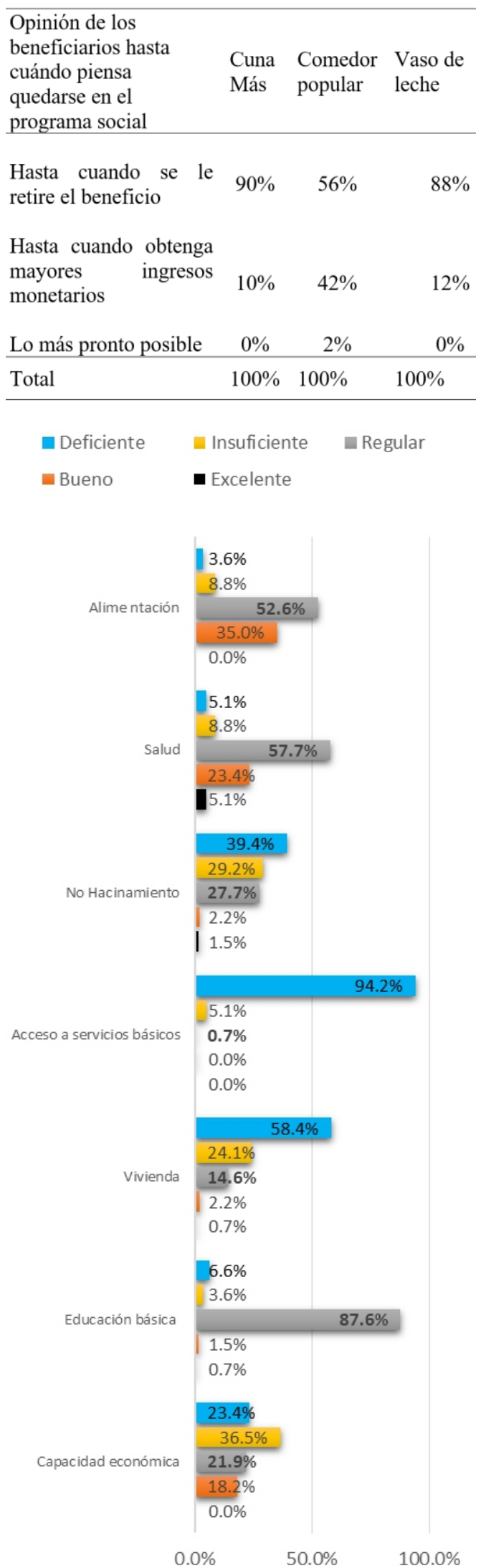

Calificación en porcentaje de Necesidades Básicas satisfechas

Figura 1: Porcentaje de calificaciones de las necesidades básicas satisfechas de los beneficiarios de los programas Vaso de Leche, Cuna Más y Comedor popular .

\begin{tabular}{lc}
\hline Línea de pobreza monetaria & $\begin{array}{c}\text { Porcentaje de } \\
\text { beneficiarios de } \\
\text { programas sociales } \\
\text { según clasificación } \\
\text { de pobreza }\end{array}$ \\
\hline $\begin{array}{l}\text { Pobre extremo (ingresos } \\
\text { menores de S/ 176) }\end{array}$ & $23,4 \%$ \\
$\begin{array}{l}\text { Pobre (ingresos entre S/176 } \\
\text { hasta S/ 326) }\end{array}$ & $58,4 \%$ \\
$\begin{array}{l}\text { No pobre (ingresos mayores a } \\
\text { S/326) }\end{array}$ & $18,2 \%$ \\
\hline Total & $100,0 \%$ \\
\hline
\end{tabular}

\section{IV.DISCUSIÓN}

Según los datos de la tabla 1 , se puede observar que la mayoría de encuestados le dan una calificación de bueno (73\%) y excelente (25\%) al programa Cuna Más. Asimismo al programa Comedor Popular recibió la calificación de bueno $(87 \%)$ y excelente (9\%). Sin embargo el programa Vaso de Leche recibió la menor calificación con bueno $(0 \%)$, excelente $(0 \%)$, y un reporte de regular $(68 \%)$ e insuficiente (30\%).

En la tabla 2, se puede observar que la mayoría de encuestados (100\%) indican que los programas sociales Cuna Más y Comedor Popular sí ayudan a reducir su pobreza. Por el contrario la mayoría $(72 \%)$ de beneficiarios del programa social Vaso de Leche indicaron que no les ha ayudado a la reducción de su pobreza.

Teniendo en cuenta los datos de la tabla 3 , se puede observar que la mayoría de encuestados Cuna Más (90\%), Comedor Popular (50\%), Vaso de leche (88\%) indican que seguirán en los programas hasta cuando se les retire el beneficio y en menor medida Cuna Más (10\%), Comedor Popular (46\%), Vaso de Leche $(12 \%)$ indican que seguirán en ellos hasta cuando obtenga mayores ingresos monetarios.

Según la figura 1 , se puede observar que la mayoría (94,4\%) de encuestados le dio una calificación de deficiente a el acceso a los servicios básicos (agua y saneamiento), seguido por la necesidad de vivienda $(58,4 \%)$ y la menor deficiencia $(3,6 \%)$ en alimentación, asignándolos a la categoría de pobres por Necesidades Básicas Insatisfechas (NBI). 
Teniendo en cuenta los datos de la tabla 4 , se puede observar que la mayoría $(58,4 \%)$ de encuestados de acuerdo a las líneas de pobreza monetaria son considerados pobres. Asimismo pobres extremos $(23,4 \%)$ y en menor medida no pobre $(18,2 \%)$.

La mayor calificación del Programa Cuna Más en comparación con el programa Comedor Popular y Vaso de Leche es concordante con los resultados de Boyd y Rentería (2018) que en su investigación "Economía del cuidado, desigualdades de género y participación en el mercado laboral: el caso de cuna más explica muestran que Cuna Más" muestran que incrementó en 14\% la probabilidad de que las usuarias estuvieran ocupadas en el mercado de trabajo (de pertenecer a la PEA ocupada).Por lo cual sería favorable en la generación de ingresos.

Se llega a resultados parecidos a Girbés (2014) con su estudio "el contrato de inclusión dialógica: una actuación de éxito en la superación de la pobreza y la exclusión social en contextos urbanos", donde indica que es importante la participación activa de los involucrados para el éxito de las políticas públicas. Este es un importante aporte ya que según los resultados el programa Vaso de Leche genera una ayuda mínima a la población y es categorizada por ellos de deficiente, sin embargo ha estado funcionando por muchos años seguidos, sin lograr mejoras considerables y aun así no se ha implementado cambios en su aplicación.

Según los resultados obtenidos de la encuesta, se concuerda con Muñoz (2016) en su investigación, "Implementación de políticas de superación de la pobreza en Chile 1990-2010. Caso Fosis”, concluyó que los altos niveles de pobreza, dan cuenta que las políticas sociales tradicionales no han sido suficientes para poder reducir la pobreza, lo que lleva a buscar nuevas formas de enfrentar el tema de la pobreza, con una mirada integral.

\section{CONCLUSIONES}

El programa Cuna Más es el más valorado por parte de los beneficiarios, con calificación de bueno (73\%) y excelente $(25 \%)$.

El programa Vaso de Leche es el único que sus beneficiarios indican que no les ha ayudado a la reducción de su pobreza $(72 \%)$.

Los beneficiarios que continuarán hasta que se les retire el beneficio son: Cuna Más (90\%), Comedor Popular (50\%), Vaso de leche (88\%).

La pobreza por necesidades básicas insatisfechas es alta $(94,4 \%)$ en la deficiencia de servicios básicos y en vivienda $(58,4 \%)$.

La pobreza monetaria es alta $(58,4 \%)$ en la clasificación pobre y en menor medida $(23,4 \%)$ como pobre extremo.

\section{REFERENCIAS BIBLIOGRÁFICAS}

Banco Mundial (2017). Informe anual 2017. $\mathrm{R}$ e c u p e r a d o d e https://openknowledge.worldbank.org/bitstr eam/handle/10986/27986/211119SP.pf

Bugallo, C. (2015). ¿Qué es el análisis de las políticas pú b l i c a s ? R e c u p e r a d o d e http://www.elsalmoncontracorriente.es/?Qu e-es-el-analisis-de-las

Boyd, C y Rentería, J. (2018). Economía del cuidado, desigualdades de género y participación en el mercado laboral: el caso de cuna más explica muestran que Cuna Más. Recuperado de https://www.cies.org.pe/sites/default/files/in vestigaciones/tercer_informe_final__cuna_mas_cies_12032018_cb_jm_version _final_pdf

CEPAL (2017). La pobreza aumentó en 2016 en América Latina y alcanzó al 30,7\% de su población, porcentaje que se mantendría estable en 2017. Recuperado de https://www.cepal.org/es/comunicados/lapobreza-aumento-2016-america-latinaalcanzo-al-307-su-poblacion-porcentajeque-se

ENCOVI (2017). Radiografía de la crisis venezolana. $\mathrm{R} \mathrm{e} \mathrm{c} \mathrm{u} \mathrm{p} \mathrm{e} \mathrm{r} \mathrm{a} \mathrm{d} \mathrm{o} \mathrm{d} \mathrm{e}$ http://elucabista.com/2018/02/21/resultados -encovi-2017-radiografia-la-crisisvenezolana/

El Informante (2016). Son 5 los distritos más pobres de la región Lambayeque. Recuperado de http://www.elinformante.pe/plantilla_notici a.php? $\operatorname{codnoticia}=275 \& \operatorname{cod}=3$

DePerú (2018).Programas sociales. Recuperado de http://www.deperu.com/abc/351/programas -sociales

Girbés, S. (2014). El Contrato de Inclusión Dialógica: una actuación de éxito en la superación de la pobreza y la exclusión social en contextos urbanos. Tesis doctoral. Universidad de Barcelona. España.

INEI (2016). Informe Técnico: Evolución de la Pobreza Monetaria 2007-2016.Recuperado d 
https://www.inei.gob.pe/media/MenuRecur sivo/publicaciones_digitales/Est/Lib1425/c ap02.pdf

INEI (2017). Evolución de la pobreza monetaria en el P e rú, 2016 . R e c u p e r a d o d e https://www.inei.gob.pe/media/cifras_de_p obreza/evolucion-de-la-pobreza-2016-10de-mayo-presentacion.pdf

INEC (2018).Pobreza por necesidades básicas insatisfechas. Recuperado de http://www.ecuadorencifras.gob.ec/pobreza -por-necesidades-basicas-insatisfechas/

MEF (2018). ¿Qué programas sociales desarrolla el Estado y cómo se clasifican? Recuperado de https://www.mef.gob.pe/es/politicaeconomica-y-social-sp-2822/750preguntas-frecuentes-pol-econ/4861-94que-programas-sociales-desarrolla-elestado-y-como-se-clasifican

Ministerio de la Mujer y Poblaciones Vulnerables (2016). Intervención de los Programas Sociales del MIMP en la Región L a m b a y que. Re cuperado de https://www.mimp.gob.pe/omep/archivos/e

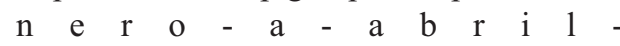
2016/BOLETINES\%20REGIONALES/bol etin_lambayeque.pd

Muñoz, C. (2016). Implementación de políticas de superación de la pobreza en Chile. Tesis de postgrado, Universidad Complutense de Madrid. España.

La República (2017). Dos de cada diez peruanos viven en condición de pobreza. Recuperado de http://larepublica.pe/economia/1040409dos-de-cada-diez-peruanos-viven-encondicion-de-pobreza

ONU (2017).17 Objetivos para Cambiar Nuestro $\mathrm{M} \mathrm{un} \mathrm{d} \mathrm{o.} \mathrm{R} \mathrm{e} \mathrm{c} \mathrm{u} \mathrm{p} \mathrm{e} \mathrm{r} \mathrm{a} \mathrm{d} \mathrm{o} \mathrm{d} \mathrm{e}$ http://www.un.org/sustainabledevelopment/ es/poverty/

RT (2016). 11 datos sobre la pobreza mundial. $\mathrm{R}$ e c u p e r a d o d e https://actualidad.rt.com/actualidad/224433 -hechos-pobreza-mundial 